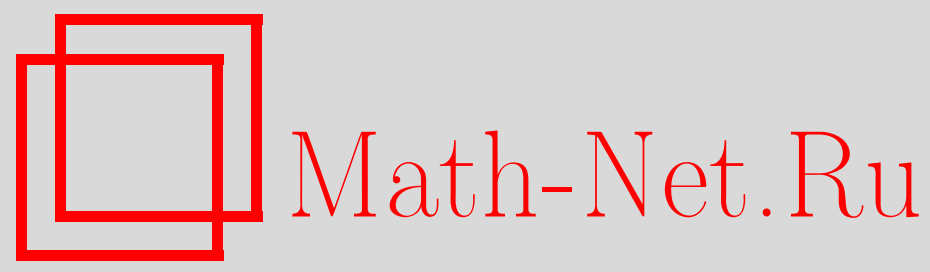

В. В. Филиппов, О пространстве оптимальных решений и о регуляризации уравнений с разрывами по пространственным переменным, Матем. заметки, 2001, том 70, выпуск 3, 458-467

DOI: https://doi.org/10.4213/mzm757

Использование Общероссийского математического портала Math-Net.Ru подразумевает, что вы прочитали и согласны с пользовательским соглашением http://www . mathnet.ru/rus/agreement

Параметры загрузки:

IP: 44.207 .124 .84

26 апреля 2023 г., 11:30:45

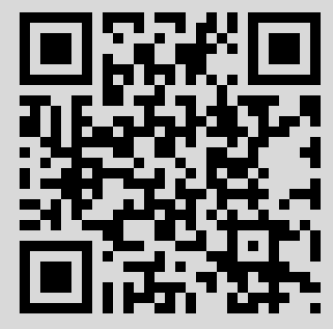




\section{О ПРОСТРАНСТВЕ ОПТИМАЛЬНЫХ РЕШЕНИЙ И О РЕГУЛЯРИЗАЦИИ УРАВНЕНИЙ С РАЗРЫВАМИ ПО ПРОСТРАНСТВЕННЫМ ПЕРЕМЕННЫМ}

\section{В. В. Филиппов}

В заметке указьвается возможность исследования свойств оптимальных решений в обход теории задачи Коши для уравнения синтеза оптимального управления.

Библиографоия: 16 названий.

В этой заметке мы обсудим возможность исследования свойств оптимальньх решений в обход теории задачи Коши для уравнения, синтезируюшего оптимальное управление.

А.В. Арутюнов обратил мое внимание на то обстоятельство, что практически нет хорошей теории задачи Коши для уравнений, возникаюших при синтезе оптимального управления. Причем это проявляется уже в линейной задаче быстрейшего попадания в начало координат.

В этой статье будет предложен нестандартньй подход к подобным ситуациям. Речь идет о том, что целью теории задачи Коши является проверка вьполнения короткого списка базовых свойств решений (теорема существования решения с данными начальными условиями, теорема о непрерьвности зависимости решений от начальных условий и т.д.). В рамках подхода [1]-[6] к теории обыкновенных дифференциальных уравнений этот список получил вполне четкие границы. При этом было показано, что можно излагать содержательную часть теории обыкновенных дифференциальных уравнений, отталкиваясь от фигурируюших в подобных списках свойств решений как от аксиом теории.

Тем самым, если мы покажем, что оптимальные решения удовлетворяют аксиомам теории [1]-[6], то нет необходимости вьписывать и исследовать уравнение синтеза оптимального управления, если целью этого исследования является дальнейшее применение к уравнению синтеза методов и фактов теории обыкновенных дифференциальных уравнений.

Здесь мы не будем углубляться в тонкости теории оптимального управления, и в основном результате мы ограничимся простейшей ситуацией, связанной с линейной задачей быстрейшего попадания в начало координат. Мы не завершаем исследование, а

Работа выполнена при поддержке Российского фонда фундаментальных исследований, грант № 98-01-00061. 
только на простейшем указанном примере показьваем возможность такого нестандартного подхода. Это приглашение к исследованиям в указанном направлении, и мы просто отмечаем их возможную содержательность.

В теории оптимального управления часто возникают ситуации, когда оптимальные решения теряют непрерывность зависимости от начальных условий. Но и в этом случае переход на уровень топологических структур [1]-[6] может помочь в исследовании. При этом имеется в виду, что реально непрерывность зависимости от начальных условий (и некоторые другие свойства решений такие, как условие Кнезера, ацикличность интегральных воронок и т.д.; см. ниже примеры 2 и 3 ) нарушается на некотором подмножестве $P$ области определения правой части. Вне множества $P$ наш подход работает в обычном режиме, а множество $P$ подлежит дальнейшему исследованию как множество особенностей, к чему наш подход хорошо приспособлен (см., в частности, последний абзац $\S 2$ в [1], где, правда, обсуждается несколько иной аспект исследования особенностей).

При исследовании уравнений синтеза оптимального управления часто пользуются “регуляризациями" (см., в частности, обсуждение этого вопроса в [7], а также [8]). В связи с этим мы предпошлем основному содержанию заметки несколько относящихся к этому вопросу примеров. Для нас важно то, что "регуляризации" могут дать не те решения, которые нужны для оптимального управления.

Наиболее простой "регуляризацией” является переход от рассматриваемого уравнения $y^{\prime}=f(t, y)$ к включению $y^{\prime} \in F(t, y)$, где $F(t, y)$ - замкнутая выпуклая оболочка предельных значений $f\left(t, y^{*}\right)$ при $y^{*} \rightarrow y$. Будем назьвать ее максимальной регуляризачией. А.Ф. Филиппов предложил свой вариант регуляризации, при котором пренебрегают значениями правой части на подмножествах меры нуль (см. точное определение в [9]). Связь между названными регуляризациями состоит в следующем: $f(t, y) \in F(t, y)$ и $F_{\varphi}(t, y) \subseteq F(t, y)$, где $y^{\prime} \in F_{\varphi}(t, y)$ - регуляризация по А. Ф. Филиппову. Четкой обязательной связи между значениями $f(t, y)$ и $F_{\varphi}(t, y)$ при этом нет. Соответственно для пространств решений имеем $D(f) \subseteq D(F)$ и $D\left(F_{\varphi}\right) \subseteq D(F)$ с аналогичным замечанием: четкой связи между $D(f)$ и $D\left(F_{\varphi}\right)$ нет. Последнее означает, в частности, что решение исходного уравнения $y^{\prime}=f(t, y)$ не обязано быть решением включения $y^{\prime} \in F_{\varphi}(t, y)$. В противоположность этому при максимальной регуляризации при переходе от уравнения $y^{\prime}=f(t, y)$ к включению $y^{\prime} \in F(t, y)$ решения не теряются: как мы отметили, $D(f) \subseteq D(F)$. Как мы увидим ниже, при любой из этих двух регуляризаций могут появиться новые решения, причем в силу отмеченного включения $D\left(F_{\varphi}\right) \subseteq D(F)$, если новые решения появились при регуляризации по А. Ф. Филиппову, то они обязательно появляются и при максимальной регуляризации, поэтому в ниже приведенных примерах, отмечая появление новых решений при регуляризации по А.. Филиппову, дальше мы уже фактически не обсуждаем этот вопрос в связи с максимальной регуляризацией.

Начнем с не связанного с управлением примера, но простого и принципиального, показывающего, как меняются решения при "регуляризации" уравнений с разрывами по пространственным переменным.

ПримеР 1. Множество $\mathbb{Q}$ рациональных чисел счетно. Занумеруем произвольньм образом его элементы: $\mathbb{Q}=\left\{x_{i}: i=1,2, \ldots\right\}$. При любом $k=1,2, \ldots$ мера множества

$$
U_{k}=\cup\left\{\left[x_{i}-2^{-(i+k+1)}, x_{i}+2^{-(i+k+1)}\right]: i=1,2, \ldots\right\}
$$


не превосходит $2^{-k}$. Имеем включения $\mathbb{R} \supseteq U_{1} \supseteq U_{2} \supseteq \cdots$. Функция

$$
f(t)= \begin{cases}1, & \text { если } t \in\left(\mathbb{R} \backslash U_{1}\right) \cup\left(\cap\left\{U_{i}: i=1,2, \ldots\right\}\right), \\ 2-2^{-i}, & \text { если } t \in U_{i} \backslash U_{i+1}, i=1,2, \ldots,\end{cases}
$$

измерима и принимает значения в отрезке $[1,2]$. Дифференциальное уравнение $y^{\prime}=f(y)$ попадает в сферу приложений нашей теории (в частности, для него справедливы теоремы существования решения задачи Коши и о непрерывности зависимости решений задачи Коши от начальньх условий; см. [4, пример 12.3.1] или [5]). И при максимальной регуляризации, и при регуляризации по А.Ф. Филиппову [9] мы переходим к соответствующему дифференциальному включению $y^{\prime} \in F(y)$, для которого $2 \in F(y)$ при любом $y \in \mathbb{R}$. Это означает, что функция $y(t)=2 t$ является решением включения $y^{\prime} \in F(y)$. Но очевидно, при обычном (восходящем к Каратеодори) определении решения эта функция не является решением исходного уравнения $y^{\prime}=f(y)$.

Перейдем к уравнениям с разрывами по пространственньп переменным, связанньми с синтезом оптимального управления. Чтобы избавить себя от несущественных в контексте обсуждаемого вопроса технических деталей, мы будем обсуждать его на уровне дифференциальных включений (т.е. забудем про управление). Имея целью первый раз обрисовать возникающие связи с подходом [1]-[6], ограничимся рассмотрением простейших ситуаций. Ограничимся также рассмотрением только одной задачи, задачи быстрейшего попадания в начало координат.

ПримеР 2. На плоскости $U=\mathbb{R} \times \mathbb{R}$ рассмотрим дифференциальное включение $y^{\prime} \in F(y)$, где для $y=(s, t)$

$$
F(s, t)= \begin{cases}{[-2,2] \times[-2,2],} & \text { если }|s|=|t|, \\ {[-1,1] \times[-1,1]} & \text { в противном случае. }\end{cases}
$$

Рассмотрим в связи с ним задачу быстрейшего попадания в начало координат. Как легко видеть, траектория оптимального решения, выходя из произвольной точки плоскости, спускается сначала по перпендикуляру к ближайшей биссектрисе одного из координатных углов, а затем движется по этой биссектрисе к началу координат. Причем этот путь проходится с максимально возможными скоростями. То есть решения указанной задачи оптимизации совпадают с решениями дифференциального включения $y^{\prime} \in f(y)$, где для $y=(s, t)$ имеем

$$
f(s, t)= \begin{cases}\{0,0\}, & \text { если } s=t=0, \\ \{(-2 \operatorname{sgn} s,-2 \operatorname{sgn} t)\}, & \text { если }|s|=|t|>0, \\ \{(-1,1)\}, & \text { если } 0<t<s \text { или } t<s<0, \\ \{(1,-1)\}, & \text { если } t>s>0 \text { или } s<t<0, \\ \{(-1,-1)\}, & \text { если }-s<t<0 \text { или } t>-s>0, \\ \{(1,1)\}, & \text { если } t<-s<0 \text { или } 0<t<-s, \\ \{(-1,1),(-1,-1)\}, & \text { если } s>0, t=0, \\ \{(1,1),(1,-1)\}, & \text { если } s<0, t=0, \\ \{(1,-1),(-1,-1)\}, & \text { если } s=0, t>0, \\ \{(1,1),(-1,1)\}, & \text { если } s=0, t<0 .\end{cases}
$$


Посмотрим, что дает регуляризация по А. Ф. Филиппову (см. [9]) в точках биссектрис координатных углов. Для определенности ограничимся биссектрисой первого координатного угла, т.е. будем предполагать, что $s=t>0$. На дополнении к биссектрисе в малой окрестности рассматриваемой точки правая часть непрерывна, и поэтому регуляризация ничего здесь не меняет. Перейдем к самой биссектрисе. При регуляризации по А.. . Филиппову мы пренебрегаем поведением правой части на подмножествах меры нуль. Тем самым, мы можем отбросить значения правой части на самой биссектрисе. Беря вьпуклую оболочку предельных значений правой части по дополнению к биссектрисе, получаем отрезок на плоскости с концами $(-1,1)$ и $(1,-1)$. Но траектория не может покинуть биссектрису. Единственной же точкой указанного отрезка, обеспечивающей движение вдоль биссектрисы, является точка $(0,0)$. То есть, дойдя до биссектрисы, решения регуляризованного уравнения останавливаются. Решения исходной задачи оптимизации потеряны. При максимальной регуляризации мы не теряем оптимальные решения, но новые "неоптимальные" решения все равно приобретаем. Что касается нашего подхода к исследованию уравнений, здесь уравнение подпадает под действие наших методов в качестве простейшего случая.

Перейдем теперь к точкам осей координат. Для определенности ограничимся положительным лучом оси абсцисс, т.е. будем предполагать, что $s>0, t=0$. Повторяя процедуру, описанную в предыдущем абзаце, получаем в качестве результата регуляризации отрезок на плоскости с концами $(-1,1)$ и $(-1,-1)$. В частности, мы получаем возможность двигаться вдоль оси абсцисс с вектором скорости $(-1,0)$, а это не имеет отношения к исходной задаче оптимизации. В исследуемых точках не вьполнено условие Кнезера (см. [2, § VII.1] или [4, § 8.1]), поэтому предложенные в [5], [10], [11] методы исследования уравнений с разрьвами по пространственным переменным не работают здесь в полном объеме по принципиальным соображениям. Тем не менее для нашего общего топологического подхода [1]-[4] это обычньй предмет исследования.

В разобранном примере правая часть дифференциального включения не является непрерьвной. Она только полунепрерывна сверху. Слегка усложнив техническую сторону, построим соответствующий пример с непрерывной (относительно метрики Хаусдорфа) правой частью.

ПримеР 3. На плоскости $U=\mathbb{R} \times \mathbb{R}$ рассмотрим дифференциальное включение $y^{\prime} \in f(y)+H$, где $f(s, t)=\left(-s t^{2},-s^{2} t\right)$ для $y=(s, t)$ и

$$
H=\{(s, t): s, t \in \mathbb{R},|s| \leqslant 1,|t| \leqslant 1\} .
$$

Как и предыдущем примере, рассмотрим задачу быстрейшего попадания в начало координат. На любой прямой вида $s+t=c$, где $c \in \mathbb{R}$, максимум значения длины $s t^{2}+s^{2} t=c s t$ проекции вектора $f(s, t)$ на направление вектора $(-1,-1)$ достигается в точке $s=t=c / 2$, поэтому траектория быстрейшего спуска для точек биссектрисы первого координатного угла идет по самой биссектрисе. При этом в $H$ надо взять значение $(-1,-1)$. Ситуация в точках других координатных углов аналогична. При этом для точек второго квадранта в $H$ надо взять значение $(1,-1)$, для точек третьего квадранта надо взять значение $(1,1)$, для точек четвертого квадранта надо взять значение $(-1,1)$.

Дополнение к биссектрисам координатных углов распадается в объединение четырех углов, стороны которых идут по этим самым биссектрисам. Чтобы понять, что происходит там, рассмотрим угол между биссектрисами первого и второго координатных 
углов. В нем приближение к оси абсцисс идет тем быстрее, чем дальше мы находимся от оси ординат, поэтому в качестве значения в $H$ мы должны брать тот из углов квадрата $H$, который смотрит в сторону ближайшей биссектрисы. Ситуация в остальных углах аналогична.

Более точно это рассуждение можно провести, опираясь на теорему сравнения (см. $[12])$.

Сказанное означает, что решения рассматриваемой задачи оптимизации совпадают с решениями дифференциального включения $y^{\prime} \in g(y)$, где для $y=(s, t)$ имеем

$$
g(s, t)=f(s, t)+ \begin{cases}\{0,0\}, & \text { если } s=t=0, \\ \{(-\operatorname{sgn} s,-\operatorname{sgn} t)\}, & \text { если }|s|=|t|>0, \\ \{(-1,1)\}, & \text { если } 0<t<s \text { или } t<s<0, \\ \{(1,-1)\}, & \text { если } t>s>0 \text { или } s<t<0, \\ \{(-1,-1)\}, & \text { если }-s<t<0 \text { или } t>-s>0, \\ \{(1,1)\}, & \text { если } t<-s<0 \text { или } 0<t<-s, \\ \{(-1,1),(-1,-1)\}, & \text { если } s>0, t=0, \\ \{(1,1),(1,-1)\}, & \text { если } s<0, t=0, \\ \{(1,-1),(-1,-1)\}, & \text { если } s=0, t>0, \\ \{(1,1),(-1,1)\}, & \text { если } s=0, t<0 .\end{cases}
$$

В результате мы получили уравнение с разрьвной правой частью. В смысле общих наблюдений ситуация здесь аналогична тому, что мы имели в предыдущем случае. Вне осей координат и биссектрис координатных углов правая часть этого дифференциального уравнения непрерывна. Регуляризация по А.. Филиппову дает дифференциальное включение $y^{\prime} \in G(y)$, где для $y=(s, t)$ обозначено $G(s, t)=f(s, t)+L$ и $L$ есть

$$
\begin{aligned}
& \text { точка }\{(-1,1)\} \text {, если } 0<t<s \text { или } t<s<0 ; \\
& \text { точка }\{(1,-1)\} \text {, если } t>s>0 \text { или } s<t<0 ; \\
& \text { точка }\{(-1,-1)\} \text {, если }-s<t<0 \text { или } t>-s>0 ; \\
& \text { точка }\{(1,1)\} \text {, если } t<-s<0 \text { или } 0<t<-s ; \\
& \text { отрезок на плоскости, соединяющий точки }(0,-1) \text { и }(0,1) \text {, если } s \neq 0, t=0 ; \\
& \text { отрезок на плоскости, соединяющий точки }(-1,0) \text { и }(0,1) \text {, если } s=0, t \neq 0 ; \\
& \text { отрезок на плоскости, соединяющий точки }(-1,1) \text { и }(1,-1) \text {, если } s=t \neq 0 ; \\
& \text { отрезок на плоскости, соединяющий точки }(1,-1) \text { и }(-1,1) \text {, если } s=-t \neq 0 \text {; } \\
& \text { квадрат } H \text { (с вершинами }(1,1),(-1,1),(-1,-1),(1,-1)) \text {, если } s=t=0 .
\end{aligned}
$$

При этой регуляризации в точках биссектрис координатных углов и на координатных осях происходит то же, что происходило в предыдущем примере, а именно: какие-то оптимальные решения пропадают и появляются какие-то новые решения, уже не оптимальные.

Тем самым, регуляризации не оставляют здесь возможности дальнейшего исследования оптимальных решений. Что касается нашей общей теории [1]-[4], мы не покидаем ее рамок.

Покажем теперь, как возникают объекты нашей абстрактной теории при рассмотрении оптимальных решений. Как и раньше, будем рассматривать задачу быстрейшего попадания в начало координат. 
ПРЕДПОЛОЖЕНИЕ А. Пусть $H$ - вьпуклое компактное подмножество пространства $\mathbb{R}^{n}$, содержащее внутри себя начало координат $\overrightarrow{0}$, функция $f: \mathbb{R}^{n} \rightarrow \mathbb{R}^{n}$ локально удовлетворяет условию Липшица, ни одно решение дифференциального включения $y^{\prime} \in f(y)+H$ не уходит на бесконечность за конечное время, $f(\overrightarrow{0})=\overrightarrow{0}$, для любой точки $y_{0} \in \mathbb{R}^{n}$ существует траектория рассматриваемого включения, начинающаяся в $y_{0}$ и заканчивающаяся в $\overrightarrow{0}$.

В предположениях $\mathbf{A}$ пространство $D(f+H)$ решений дифференциального включения $y^{\prime} \in f(y)+H$ принадлежит классу $R_{c e}(U)$, где $U=\mathbb{R} \times \mathbb{R}^{n}$ (см. [1]-[6]).

Обозначим через $\Phi$ множество решений указанной задачи для дифференциального включения $y^{\prime} \in f(y)+H$. Если $\varphi \in \Phi, \pi(\varphi)=[a, b]$ и $c \in[a, b]$, то $\left.\varphi\right|_{[c, b]} \in \Phi$. Множество $Z$ всевозможных ограничений элементов $\Phi$ на отрезки, лежащие в их областях определения, принадлежит классу $R^{i}(U)$. Кроме того, взаимноепродолжение двух элементов из $Z$, определенных на двух смежньх отрезках, с совпадаюшим значением в общей точке их областей определения также принадлежит $Z$. Таким образом, $Z \in R(U)$. То есть множество $Z$ оптимальных решений обладает требуемой комбинаторной структурой.

Для $y \in U$ обозначим через $\sigma(y)$ множество всех решений $z$ дифференциального включения $y^{\prime} \in f(y)+H$, для которых $\inf \pi(z)=0, y(0)=y, z(\sup \pi(z))=\overrightarrow{0}$ и $z(t) \neq \overrightarrow{0}$ при $t<\sup \pi(z)$. Пусть $\tau(y)=\inf \{\sup \pi(z): z \in \sigma(y)\}$ (в сделанных предположениях множество $\sigma(y)$ непусто).

Покажем, что в предположениях $\mathbf{A} \tau(y)=\sup \pi(z)(<\infty)$ при некотором $z \in \sigma(y)$. Для этого зафиксируем произвольную последовательность $\left\{z_{i}: i=1,2, \ldots\right\} \subseteq \sigma(y)$, для которой $\sup \pi\left(z_{i}\right) \rightarrow \tau(y)(<\infty)$. При некотором $M<\infty$ включение $\pi\left(z_{i}\right) \subseteq[0, M]$ имеет место при всех $i=1,2, \ldots$, поэтому в силу того, что ни одно решение дифференциального включения $y^{\prime} \in f(y)+H$ не уходит на бесконечность за конечное время, из [2, лемма VI.2.1] или из [4, лемма 7.2.1] вытекает равномерная ограниченность решений $z_{i}, i=1,2, \ldots$. В силу условия $(c)$ отсюда вытекает, что из последовательности $\left\{z_{i}: i=1,2, \ldots\right\}$ можно выбрать сходящуюся подпоследовательность. Ее предел $z$ является искомым решением, что и дает требуемое. При этом $z \in \Phi$.

Чтобы иметь возможность использовать по существу аппарат теории [1]-[4], мы должны прежде всего получить соответствующий результат о непрерывности зависимости решений от правой части. Сделаем это.

УТВЕРЖДЕНИЕ 1. Пусть при любом $i=0,1,2, \ldots$ функиии $f_{i}: \mathbb{R}^{n} \rightarrow \mathbb{R}^{n}$ и компакты $H_{i}$ удовлетворяют условию $\mathbf{A}($ в качестве $f$ и $H)$. Пусть последовательность функиий $\left\{f_{i}: i=1,2, \ldots\right\}$ сходится $\kappa$ функиии $f_{0}$ равномерно на любом компактном подмножестве пространства $\mathbb{R}^{n}$, а последовательность компактов $\left\{H_{i}: i=1,2, \ldots\right\}$ сходится $\kappa$ компакту $H$ относительно метрики Хаусдорфа. Пусть $Z_{i}$ обозначает пространство оптимальных решений включения $y^{\prime} \in f_{i}(y)+H_{i}$. Тогда последовательность пространств $\left\{Z_{i}: i=1,2, \ldots\right\}$ сходится в $U \kappa$ пространству $Z_{0}$.

ДокАЗАТЕЛЬСТво. 1) Пусть $C$ - бесконечное подмножество натурального ряда, $K \subseteq \mathbb{R} \times \mathbb{R}^{n}$ - произвольный компакт. Пусть $z_{i} \in\left(Z_{i}\right)_{K}$ для $i \in C$. Наша цель - показать, что из последовательности $\left\{z_{i}: i \in C\right\}$ можно выбрать подпоследовательность, сходящуюся к некоторой функции из $Z_{0}$. 
Для $i=1,2, \ldots$ и $y \in \mathbb{R}^{n}$ определим $\Phi_{i}, \sigma_{i}(y)$ и $\tau_{i}(y)$ по $f_{i}$ и $H_{i}$ аналогично тому, как выше мы определяли $\Phi, \sigma(y)$ и $\tau(y)$ по $f$ и $H$.

2) Без ущерба для общности мы можем предположить дополнительно, что в 1) последовательность $\left\{z_{i}\left(\inf \pi\left(z_{i}\right)\right): i \in C\right\}$ сходится к некоторой точке $y_{0} \in \mathbb{R}^{n}$.

Зафиксируем функцию $z_{0} \in \sigma_{0}\left(y_{0}\right)$, для которой $\tau_{0}\left(y_{0}\right)=\sup \pi\left(z_{0}\right)$, и для $t \in \pi\left(z_{0}\right)$ положим $\mu_{0}(t)=y_{0}^{\prime}(t)-f_{0}\left(y_{0}(t)\right)\left(\in H_{0}\right)$. Для $t>\sup \pi\left(z_{0}\right)$ положим $\mu_{0}(t) \equiv \overrightarrow{0}$. Пусть для $i=1,2, \ldots$ и $t \geqslant 0$ символ $\mu_{i}(t)$ обозначает точку компакта $H_{i}$, ближайшую к точке $\mu_{0}(t)$. Функция $\mu_{i}(t)$ измерима, это легко вьводится из утверждений в $[2$, $\S \mathrm{IV} .11, \S \mathrm{IV} .12]$ или в $[4, \S 4.12, \S 4.13]$. В силу сходимости $H_{i} \rightarrow H_{0}$ в метрике Хаусдорфа имеем сходимость $\mu_{i}(t) \rightarrow \mu_{0}(t)$ при всех $t \geqslant 0$.

Пусть для $i \in C$ символ $x_{i}$ обозначает максимально продолженное вправо решение задачи Коши $y(0)=z_{i}\left(\inf \pi\left(z_{i}\right)\right)$ для уравнения $y^{\prime}=f_{i}(y)+\mu_{i}(t)$.

В силу теоремы о непрерывности зависимости решений задачи Коши от правой части и от начальных условий (см., например, [5, теорема 4.1] или [4, теорема 12.2.3]) и теоремы о единственности решения задачи Коши имеем сходимость $x_{i}\left(\sup \pi\left(z_{0}\right)\right) \rightarrow$ $z_{0}\left(\sup \pi\left(z_{0}\right)\right)=\overrightarrow{0}$.

Так как $f_{i}\left(x_{i}\left(\sup \pi\left(z_{0}\right)\right)\right) \rightarrow f(\overrightarrow{0})=\overrightarrow{0}, H_{i} \rightarrow H_{0}$ и $\overrightarrow{0} \in\left\langle H_{0}\right\rangle$, то найдутся $\varepsilon>0$ и $i_{0}=1,2, \ldots$ такие, что $O_{\varepsilon} \overrightarrow{0} \subseteq f_{i}(y)+H_{i}$ для всех $y \in O_{\varepsilon} \overrightarrow{0}$ и $i=i_{0}, i_{0}+1, i_{0}+2, \ldots \mathrm{B}$ силу этого, в свою очередь, $\tau_{i}\left(x_{i}\left(\sup \pi\left(z_{0}\right)\right)\right) \rightarrow 0$. В силу неравенства

$$
\tau_{i}\left(z_{i}\left(\inf \pi\left(z_{i}\right)\right)\right) \leqslant \sup \pi\left(z_{0}\right)+\tau_{i}\left(x_{i}\left(\sup \pi\left(z_{0}\right)\right)\right)
$$

сходимость $\tau_{i}\left(x_{i}\left(\sup \pi\left(z_{0}\right)\right)\right) \rightarrow 0$ влечет оценку

$$
\limsup _{i \in C, i \rightarrow \infty} \tau_{i}\left(z_{i}\left(\inf \pi\left(z_{i}\right)\right)\right) \leqslant \sup \pi\left(z_{0}\right)=\tau\left(y_{0}\right)
$$

3) Пусть $\varphi_{i} \in \sigma_{i}\left(z_{i}\left(\inf \pi\left(z_{i}\right)\right)\right) \cap \Phi_{i}-$ продолжение функции $z_{i}$ вправо. При этом $\sup \pi\left(\varphi_{i}\right)=\tau_{i}\left(z_{i}\left(\inf \pi\left(z_{i}\right)\right)\right)$. В силу 2$)$

$$
\limsup _{i \in C, i \rightarrow \infty} \sup \pi\left(\varphi_{i}\right) \leqslant \tau\left(y_{0}\right)
$$

поэтому найдется число $M>0$ такое, что $\sup \pi(\varphi) \leqslant M$ при всех $i \in C$. Теперь из невыхода решений включения $y^{\prime} \in f_{0}(y)+H_{0}$ на бесконечность за конечное время и сходимости $D\left(f_{i}+H_{i}\right) \rightarrow D\left(f_{0}+H_{0}\right)$ следует равномерная ограниченность функций $\varphi_{i}$, $i \in C$ (см. [2, $\S \mathrm{VI} .2]$ или $[4, \S 7.2])$. Отсюда в силу сходимости $D\left(f_{i}+H_{i}\right) \rightarrow D\left(f_{0}+H_{0}\right)$ следует, что из последовательности $\left\{\varphi_{i}: i \in C\right\}$ можновыбрать подпоследовательность $\left\{\varphi_{i}: i \in C_{1}\right\}$, сходящуюся к некоторой функции $\varphi_{0} \in D\left(f_{0}+H_{0}\right)$. Так как

$$
\begin{gathered}
\lim _{i \in C, i \rightarrow \infty} \sup \pi\left(\varphi_{i}\right)=\sup \pi\left(\varphi_{0}\right), \\
\varphi_{0}\left(\sup \pi\left(\varphi_{0}\right)\right)=\lim _{i \in C, i \rightarrow \infty} \varphi_{i}\left(\sup \pi\left(\varphi_{i}\right)\right)=\overrightarrow{0},
\end{gathered}
$$

то $\sup \pi\left(\varphi_{0}\right) \geqslant \tau\left(y_{0}\right)$. Но из первого замечания этого пункта вытекает, что

$$
\limsup _{i \in C_{1}, i \rightarrow \infty} \sup \pi\left(\varphi_{i}\right) \leqslant \tau\left(y_{0}\right)
$$


следовательно, $\sup \pi\left(\varphi_{0}\right) \leqslant \tau\left(y_{0}\right)$. Таким образом, $\sup \pi\left(\varphi_{0}\right)=\tau\left(y_{0}\right)$. В силу сходимости $D\left(f_{i}+H_{i}\right) \rightarrow D\left(f_{0}+H_{0}\right)$ из последовательности $\left\{z_{i}: i \in C_{1}\right\}$ можно выбрать подпоследовательность $\left\{z_{i}: i \in C_{2}\right\}$, сходящуюся к некоторой функции $z_{0} \in D\left(f_{0}+H_{0}\right)$. В силу сходимости последовательности $\left\{\varphi_{i}: i \in C_{1}\right\}$ к функции $\varphi_{0}$ функция $z_{0}$ является ограничением функции $\varphi_{0}$, поэтому $z_{0} \in Z_{0}$. Утверждение доказано.

Применяя доказанное утверждение к стационарным последовательностям $f_{i} \equiv f$ и $H_{i} \equiv H$, получаем

СледСтвИЕ. В предположсениях $\mathbf{A}$ пространство оптимальных решений включения $y^{\prime} \in f(y)+H$ принадлежит классу $R_{c}(U)$.

Тем самьм, в предположениях $\mathbf{A}$ мы установили наличие у пространства оптимальных решений основного топологического свойства, используемого в построениях нашей теории. То есть пространство оптимальных решений оказьвается здесь одним из возможных предметов исследования нашей общей теории. С другой стороны, сравнение результатов теории с примерами 2,3 показьвает, что при решении задачи синтеза оптимального управления не всегда получается уравнение или включение, для которого можно построить теорию на уровне классической теории обыкновенных дифференциальных уравнений. На координатных осях для пространств оптимальных решений примеров 2, 3 не справедливы ни теорема сушествования решения задачи Коши, ни теорема Кнезера. Таким образом, при исследовании таких ситуаций нет возможности использовать основные идеи классической теории обыкновенных дифференциальных уравнений, работая с "хорошими" уравнениями, синтезируюшими управление, и остается возможность вести качественные рассуждения на уровне наших пространств решений.

Иная ситуация возникает в частном случае линейной задачи.

ПрЕДПоЛОЖЕНИЕ Б. Пусть $H$ - выпуклое компактное подмножество пространства $\mathbb{R}^{n}$, содержащее внутри себя начало координат $\overrightarrow{0}$, а действительные части собственных значений квадратной матрицы $A$ порядка $n$ неположительны.

В предположениях $\mathbf{~}$ (и с $f(y)=A y$ ) для любой точки $y \in \mathbb{R}^{n}$ множество $\sigma(y) \cap \Phi$ является выпуклым. Используя этот факт, можно доказать теорему существования решения задачи Коши для оптимальных решений в полном двустороннем объеме (если оптимальное решение для данной точки есть, то мы можем уйти из точки по оптимальной траектории, но априори ничто не гарантирует нам, что мы можем войти в точку по оптимальной траектории исходной задачи).

УТВеРЖДЕнИЕ 2. В предположениях $\mathbf{5}$ пространство $Z$ оптимальных решений включения $y^{\prime} \in A y+H$ принадлежст классу $R_{c e}(U)$.

ДокАЗАТЕЛЬСТво. Принадлежность $Z \in R_{c}(U)$ уже установлена в следствии утверждения 1. Остается доказать выполнение условия $(e)$. Допустим противное. Возьмем произвольно точку $y_{0} \in \mathbb{R}^{n} \backslash\{\overrightarrow{0}\}$, в которую нельзя войти по оптимальной траектории.

Пусть $r=\frac{1}{3}\left\|y_{0}\right\|$. Найдем $\varepsilon \in(0, r)$ такое, что при $y \in O_{2 \varepsilon} y_{0}$ и $\varphi \in \sigma(y) \cap \Phi$ имеем $\varphi\left(\tau\left(y_{0}\right)\right) \in O_{r} \overrightarrow{0}$. Пусть $\Sigma=\bigcup\left\{\sigma(y) \cap \Phi:\left\|y-y_{0}\right\|=\varepsilon\right\}$. Пусть для $\varphi \in \Sigma$ и $\lambda \in[0,1]$

$$
G_{\lambda}(\varphi)=\frac{\varphi\left(\lambda \tau\left(y_{0}\right)\right)-y_{0}}{\left\|\varphi\left(\lambda \tau\left(y_{0}\right)\right)-y_{0}\right\|}
$$


Мы определили гомотопию $G_{\lambda}: \Sigma \rightarrow S$, где $S$-единичная сферапространства $\mathbb{R}^{n}$. Множество оптимальных решений $\sigma(y) \cap \Phi$, начинаюшихся в произвольной точке $y \in \mathbb{R}^{n}$, в наших предположениях выпукло, поэтому в силу теоремы Виеториса-Бигла отображение $G_{0}$ порождает изоморфизм групп рациональных гомологий. Отображение $G_{1}$ стягиваемо и поэтому порождает тривиальньй гомоморфизм групп гомологий. Так как $(n-1)$-мерная группа гомологий сферы $S$ нетривиальна, мы получаем противоречие, которое и дает требуемое. Утверждение доказано.

УТВЕРЖДЕНИЕ 3. Пусть в предположениях Б гранича компакта $H$ не содержит отрезков и каждая точка границы имеет единственную опорную плоскость. Тогда пространство $Z$ оптимальных решений включения $y^{\prime} \in A y+H$ принадлежит классу $R_{\text {сеи }}(U)$.

ДокАЗАТЕЛЬСТво сводится к соответствующим образом отредактированным выкладкам и рассуждениям из $§ 20$ и $§ 18$ книги [13].

Теорема 1. В предположениях Б пространство $Z$ оптимальных решений включения $y^{\prime} \in A y+H$ принадлежит $\left[R_{c е u}\right]_{R_{c}(U)}$.

ДокАЗАТЕЛЬСтво. Мы можем апшроксимировать в метрике Хаусдорфа любой выпуклый компакт компактами вида, указанного в утверждении 3 (см. [14], а также [15]; автор выражает благодарность С. А. Богатому за помошь в нахождении этой ссылки). Отсюда в силу утверждений 1-3 следует требуемое.

Тем самьм, в предположениях Б пространство оптимальных решений обладает всеми основными свойствами пространства решений произвольного обыкновенного дифференциального уравнения с достаточно хорошей правой частью. Это следует из апшроксимации, указанной в теореме 1: в силу изложенной в [1]-[6] теории принадлежность пространства решений классу $\left[R_{c e u}\right]_{R_{c}(U)}$ распространяет на рассматриваемое уравнение практически всю сложившуюся теорию обыкновенных дифференциальных уравнений. При этом нам фактически не нужно искать, выписьвать и исследовать уравнение, синтезирующее оптимальное управление.

В [7] рассмотрен пример линейной задачи быстрейшего попадания в начало координат, восходящий к Бруновскому [16]. В [7] четко выдержана сложившаяся идеология рассмотрения свойств оптимальных решений: сначала выписьвается уравнение, синтезирующее оптимальное управление, и уже затем, опираясь на развитую для соответствующего типа уравнений теорию задачи Коши, устанавливаются свойства оптимальных решений. Как показьвает теорема 1, нет необходимости выписьвать синтезирующее уравнение, а значительную часть информации, которую нужно извлечь из его рассмотрения в контексте обсуждаемого вопроса, можно высказать сразу. В примере из [7], [16] синтезирующее уравнение выписьвается довольно просто, как и в примеpax 1-3 этой заметки. Обычно же это довольно сложное дело.

Автор выражает благодарность А. Ф. Филиппову, А.В. Арутюнову, С. М. Асееву и С. А. Богатому за полезные обсуж дения, связанные с этой заметкой. 


\section{СПИСОК ЦИТИРОВАННОЙ ЛИТЕРАТУРЫ}

[1] Филиппов В.В. Топологическое строение пространств решений обыкновенных дифференциальных уравнений // УМН. 1993. Т. 48. № 1. С. 103-154.

[2] Филиппов В.В. Пространства решений обыкновенных дифференциальных уравнений. М.: Изд-во МГУ, 1993.

[3] Filippov V. V. Basic topological structures of the theory of ordinary differential equations // Topology in Nonlinear Analysis. Banach Center Publ. V. 35. Warsaw: Polish Acad. Sci., 1996. P. 171-192.

[4] Filippov V.V. Basic Topological Structures of Ordinary Differential Equations. Dordrecht-Boston-London: Kluwer Acad. Publ., 1998.

[5] Филиппов В.В. О теории задачи Коши для обькновенного дифференциального уравнения с разрьвной правой частью // Матем. сб. 1994. Т. 185. № 11. С. 95-118.

[6] Филиппов В.В. О гомологических свойствах множеств решений обыкновенных дифференциальных уравнений // Матем. сб. 1997. Т. 188. №6. С. 139-160.

[7] Bressan A., Colombo G. Existence and continuous dependence for discontinuous O.D.E.'s // Boll. Un. Mat. Ital. B (7). 1990. V. 4. P. 295-311.

[8] Филиппов В.В. Замечание о регуляризационной теореме А. Брессана // Дифференц. уравнения. 2000. Т. 36. № 1. С. 355-358.

[9] Филиппов А.Ф. Дифференциальные уравнения с разрьвной правой частью. М.: Наука, 1985.

[10] Филиппов В. В. Об обыкновенных дифференциальных уравнениях с разрьвными правыми частями // Дифференц. уравнения. 1994. Т. 30. № 8. С. 1299-1306.

[11] Филиппов В.В.О теории задачи Коши для обыкновенного дифференциального уравнения с разрывами по пространственным переменным // Дифференц. уравнения. 1997. Т. 33. № 7 . C. $885-891$.

[12] Филиппов В. В. О теореме сравнения // Матем. заметки. 1995. Т. 57. № 4. С. 606-624.

[13] Понтрягин Л.С., Болтянский В.Г., Гамкрелидзе Р. В., Мищенко Е.Ф. Математическая теория оптимальых процессов. М.: Наука, 1969.

[14] Klee V. Some new results on smoothness and rotundity in normed linear spaces // Math. Ann. 1959. V. 139. № 1. P. 51-63.

[15] Zamfirescu T. Nearly all convex bodies are smooth and strictly convex // Monatsh. Math. 1987. V. 103. № 1. P. 57-62.

[16] Brunovsky P. The closed-loop time-optimal control. I. Optimality // SIAM J. Control. 1974. V. 12. P. $624-634$. 